\title{
CJD variant stirs debate on release of data
}

Paris. British researchers are divided about whether there is a need to change the UK system for reporting cases of CreutzfeldtJakob disease (CJD) to provide not only numbers of confirmed cases of a novel variant of the disease as at present, but also numbers of suspected cases.

Although some argue that the early publication of such data would speed up understanding of the transmission of the disease, others fear that the information could create unnecessary panic if handled irresponsibly by the mass media.

The debate coincides with news that a 33-year-old British woman appears to be the fourteenth victim of the new variant, which is known as NV-CJD and was formally identified in March. Those suffering from it are widely considered to have contracted the disease from beef contaminated with the agent that causes bovine spongiform encephalopathy (BSE).

Further evidence to support this hypothesis comes in a paper published this week in Nature (see pages 685-690) by a research team headed by John Collinge, of Imperial College School of Medicine at St Mary's Hospital in London, indicating that the biochemical characteristics of the new variant are closer to those of BSE than those of classical CJD.

At present, the UK government releases quarterly updates of the number of 'definite and probable' cases of classic forms of CJD. But it reports only on confirmed cases of NV-CJD, usually following post-mortem neuropathology. Similarly, while the reports give the total number of 'referrals' - suspect CJD cases referred by neurologists to the National Creutzfeldt-Jakob Disease Surveillance Unit in Edinburgh - they say nothing about what proportion of these are suspected of having NV-CJD.

According to Sheila Gore, a senior statistician at the biostatistics unit in Cambridge of the Medical Research Coun- cil (MRC) and a member of the MRC's committee dealing with human spongiform encephalopathies, such information is essential if epidemiologists are to develop an early idea of changes in the incidence of the disease and of the evolution of any epidemic.

A general consensus exists among European surveillance networks, however, that distinguishing between classic CJD and the new variant among referrals would make sense only if "validated clinical criteria" were available to diagnose the new variant.

In the absence of such criteria, reporting of referrals of suspect NV-CJD cases would be "meaningless", says Robert Will, head of the UK CJD surveillance unit. He adds that the press and public might fail to make the distinction between suspect and confirmed "cases'. "Say we now had 20 suspect cases," says Will. "It may be that only three have it. To say 20 would be misleading."

Some European scientists say that Will's group does its best to make information available in the political circumstances. One adds that there is also much informal exchange of data between the UK group and other surveillance networks established under the European Union's Biomed research programme. Will himself says: "European collaboration in epidemiology may be the only solution to understanding the new variant of CJD."

Nonetheless, France decided this month to change its reporting system to provide information on suspect cases. Quarterly reports on CJD incidence will also break down the list of referred, and 'probable or confirmed', cases into seven age groups (under 30, over 80, and the intervening five 'decades'). The new reports, the first of which will be published next month, will also emphasize that many of the 'suspect' cases listed may not turn out to be CJD, much less the new variant.

The change to the French system goes further than that which Gore would like to

\section{French advisors back modified maize}

Paris. The body that advises the French government on the testing and sale of genetically modified organisms has restated its support for a bid by Monsanto to import its genetically modified maize into the European Union (EU).

The Commission du Génie Biomoléculaire described the case as an "ideal dossier" where the risks could be clearly assessed. Disagreeing with the official views of other EU countries, the commission said the maize was safe, and should be approved for sale in Europe.

The modified maize contains a gene from the bacterium Bacillus thuringiensis (which makes it resistant to the European corn borer), a herbicide-resistant gene and a marker gene conferring resistance to the antibiotic ampicillin.

The commission dismissed concern that the herbicide-resistant gene might jump to wild relatives of the crop, arguing that maize does not interbreed with other European plants. Claims that the antibiotic-resistance gene might pass to bacteria that are pathogenic to humans and animals were also discounted as "highly improbable" by the commission, which added that such genes are already ubiquitous in ruminant gut flora. D. B. see introduced in the United Kingdom. She wants a breakdown of referrals to tell how many are under the age of 40 . Cases of the new variant mainly arise in this younger age group, she argues (although not exclusively, as NV-CJD has also appeared in older patients), while classic CJD - which usually occurs in older patients - sometimes occurs in patients under 40 .

Gore claims that, whereas most referrals have previously involved patients in their sixties, the Edinburgh surveillance unit is probably "increasingly having referrals under the age of 40". Continual monitoring of the number of younger referrals and the proportion subsequently confirmed could provide useful epidemiological information, says Gore. She claims that, if a large proportion of referrals were ultimately confirmed, epidemiologists could use this ratio to make projections based on referrals without having to wait on confirmed cases.

Gore admits that such a system would only add "confusion" if the proportion was as low as 10 per cent. But she is adamant that epidemiologists should have the opportunity to establish the usefulness of data on referred cases. "We need as timely surveillance as possible," she says, arguing that information on suspect cases could provide an "earlier handle" on the evolution of any epidemic.

But Will says that calls to make the number of 'suspect' CJD cases available "reflects naivety about the difficulty of neurological diagnosis". Whereas a sound basis exists for releasing information on the total number of CJD referrals, and the number of 'probable' cases of classic forms of CJD, there is no basis for providing analogous figures specifically for the new variant, he says.

The number of referrals is meaningful for classic forms of CJD, says Will, because the latter can be fairly easily distinguished from other forms of dementia, such as Alzheimer's disease, because of the short duration of the illness and a characteristic electroencephalogram (EEG) that permits a clinical diagnosis in 60 to 70 per cent of patients. But the new variant of CJD is much more difficult to distinguish from other forms of dementia, he says, because the duration of illness is longer and the EEG provides little information. The age of patients is a poor tool for discriminating between CJD and other dementia, says Will.

Will says that the way forward is for his group to publish data on cases of NV-CJD in a peer-reviewed journal as soon as possible. Epidemiologists need not just information on incidence, but also on the age of onset of the disease, the date of referral and the date of death. "This is important epidemiological data that people should have available," he says, adding that publication of the data is imminent.

Declan Butler 